\title{
SIMULTANEOUS HPLC-UV QUANTIFICATION OF DILTIAZEM AND N-DEMETHYLDILTIAZEM IN HUMAN PLASMA
}

S. S. Hasan ${ }^{1 *}$, Nisar-ur-Rahman ${ }^{2}$, Khalid Hussain $^{1}$ and S. Atif Raza ${ }^{1}$

${ }^{1}$ University College of Pharmacy, Punjab University, Allama Iqbal Campus, Lahore-54000, Pakistan

${ }^{2}$ Department of Pharmacy, Islamia University Bahawalpur, Pakistan

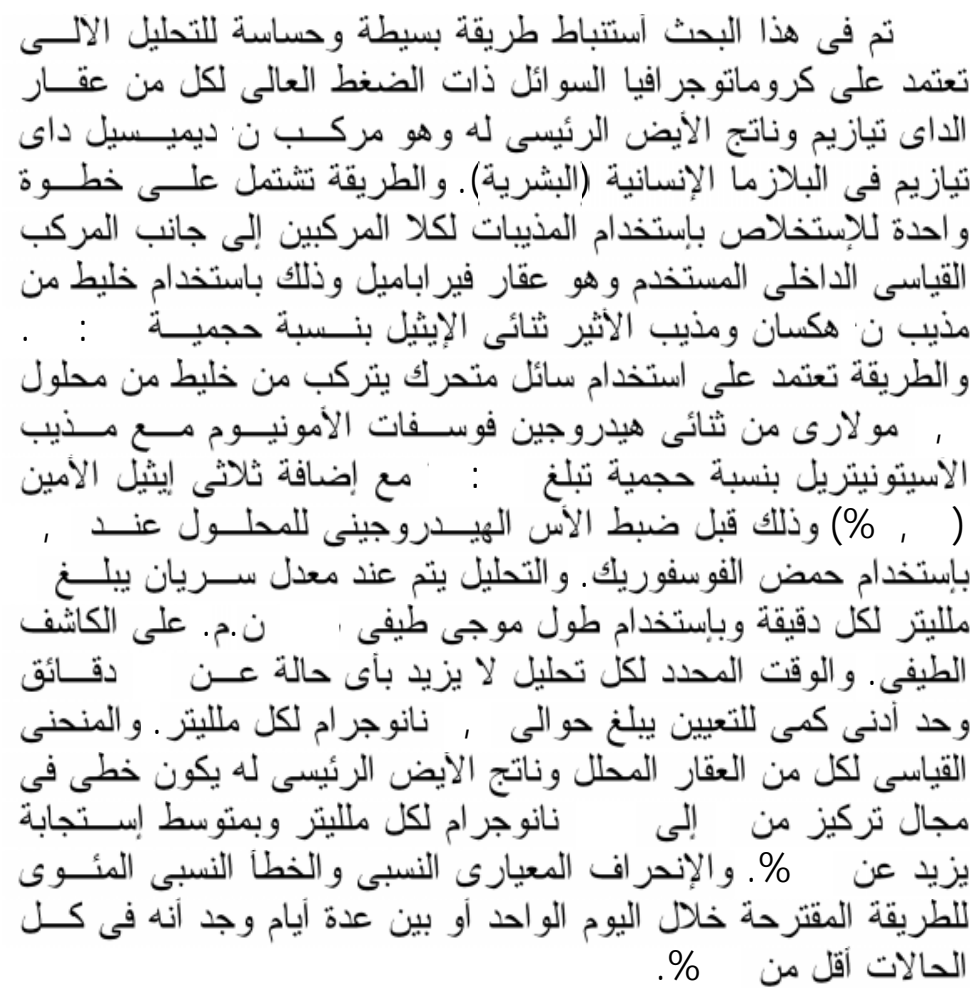

A simple and sensitive reversed phase high-performance liquid chromatographic method was developed for simultaneous quantification of diltiazem $\mathrm{HCl}$ and its major metabolite $\mathrm{N}$ demethyldiltiazem in human plasma. The method involves one step solvent extraction of diltiazem, $N$-demethyldiltiazem and the

Received in $7 / 4 / 2005$ \& Accepted in 12/9/2005

*Corresponding author 
internal standard, verapamil with $n$-hexane and diethyl ether $(50: 50 \mathrm{v} / \mathrm{v})$. The mobile phase comprised $0.1 \mathrm{M}$ ammonium dihydrogen phosphate-acetonitrile $(62: 38 \mathrm{v} / \mathrm{v})$ and triethylamine (0.08\%) was added before the $\mathrm{pH}$ was adjusted to 5.9 with $85 \%$ phosphoric acid. Analysis was run at a flow rate of $1.0 \mathrm{ml} / \mathrm{min}$ at a detection wavelength of $238 \mathrm{~nm}$. The completion time for assay was not more than 10 minutes and lower limit of quantification was 5 $\mathrm{ng} / \mathrm{ml}$. The calibration curve for diltiazem and its metabolite was linear over a concentration range of 5-200 $\mathrm{ng} / \mathrm{ml}$ and average recovery was about $90 \%$. The coefficient of variation and percent error values of the assay method within and between days were all less than $10 \%$.

\section{INTRODUCTION}

Diltiazem $\mathrm{HCl}$ is a calcium channel blocker used in the treatment of angina and also effective in the treatment of hypertension and cardiac arrhythmias. Diltiazem undergoes more extensive first pass metabolism due to the slower rate of absorption, which results in about $40 \%$ bioavailability. ${ }^{1}$ Orally administered diltiazem is metabolized into two basic active metabolites Ndemethyldiltiazem and deacetyldiltiazem. The former was reported to be the major metabolite in human and its half-life was found to be similar to that diltiazem whereas the half- life of deacetyldiltiazem was longer. ${ }^{2}$ Chemical structure of diltiazem $\mathrm{HCl}$ and its two metabolites are shown in Fig. 1.

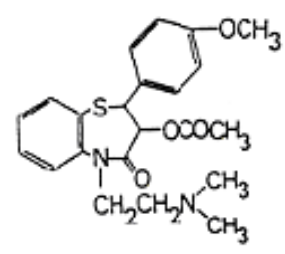

\section{Diltiazem HCl}

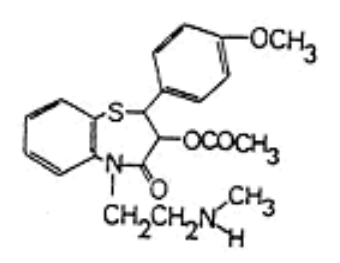

N-Demethyldiltiazem

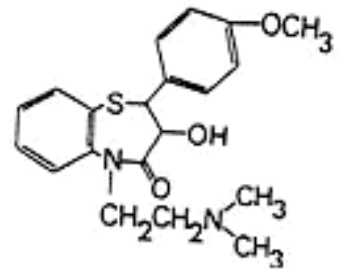

Deacetyldiltiazem

Fig.1: Chemical structures of diltiazem $\mathrm{HCl}$ and its two active metabolites. 
A review of the literature revealed that several high performance liquid chromatographic (HPLC) methods have been reported for the determination of diltiazem alone or along with its major metabolites in the human plasma. $^{3-6}$ Most of the methods involve liquid-liquid extractions with slight differences in the extraction solvent, mobile phase, the column and the run time for the analysis. Some authors employed reversed phase chromatography with either acid back-extraction ${ }^{7}$ or solidphase extraction procedure ${ }^{8}$ and a normal-phase method. ${ }^{9}$ A direct injection of plasma samples into the automatic chromatographic system for on-line clean up of plasma samples was also reported by Ascalone and Dalbo. ${ }^{10}$ Only few methods permit separation and quantification of both diltiazem and its active metabolites in human plasma. After oral administration of diltiazem, the blood plasma concentration of $\mathrm{N}$-demethyldiltiazem compared to deacetyldiltiazem rises with time, ${ }^{11}$ which should be quantified along with diltiazem.

In this paper, we report a simple, sensitive and specific HPLC method for routine quantification of not only diltiazem but also its major metabolite, $\mathrm{N}$-demethyldiltiazem in human plasma using ultraviolet detection. The assay method was evaluated for accuracy, precision, recovery and linearity. The method employed a mobile phase of $\mathrm{pH}$ 5.9, permitting the use of a cheaper silicabased column. Also, a widely available compound, verapamil was used as the internal standard. The method was developed in order to support human pharmacokinetic/ bioavailability studies following the administration of a sustained release pellets containing $90 \mathrm{mg}$ diltiazem $\mathrm{HCl}$.

\section{EXPERIMENTAL}

\section{Materials}

Diltiazem hydrochloride (Sigma, USA), verapamil hydrochloride (USP, Reference standard), Ndemethyldiltiazem (Tanabe, Japan), acetonitrile, HPLC grade (Malinckrodt, USA), diethyl ether, AR (BDH, England), N-hexane, AR (Malinckrodt, USA), ammonium dihydrogen ortho phosphate, AR (BDH, England), triethylamine, AR (Fluka, Switzerland) and phosphoric acid, AR (BDH, England).

\section{Instrumentation}

The HPLC system consisted of a Jasco PU-980 Intelligent HPLC pump, a Gilson 119 UV/VIS detector (Gilson Medical Electronics, Villiersle-Bel, France) connected to a Hitachi D-2500 integrator (Hitachi, Tokyo, Japan) and a Rheodyne 7125 sample injector fitted with a $50 \mu \mathrm{l}$ sample loop. The detector was operated using a sensitivity range of 0.005 AUFS and wavelength of $238 \mathrm{~nm}$. A LiChrospher 100 RP-18e reversed phase column $(5 \mu \mathrm{m}, 250-\mathrm{x} 4.6 \mathrm{~mm}$ ID) (Merck, Germany) fitted with a refillable guard column was used for the separation. The mobile phase 
comprised $\quad 0.1 \mathrm{M} \quad$ ammonium dihydrogen phosphate and acetonitrile $(62: 38 \mathrm{v} / \mathrm{v})$. Triethylamine $(0.08 \%)$ was added before the adjustment of $\mathrm{pH}$ to 5.9 with $85 \%$ phosphoric acid and filtered through a $0.45 \mu \mathrm{m}$ Millipore filter. The samples were centrifuged in a Labofuge 200 centrifuge (Heraeus Sepatech, Germany). Analysis was run at a flow rate of $1.0 \mathrm{ml} / \mathrm{min}$ and the detection wavelength was $238 \mathrm{~nm}$ with a sensitivity range of 0.005 aufs.

\section{Standard solutions}

Stock solutions were prepared by dissolving $100 \mathrm{mg}$ of diltiazem or $\mathrm{N}$ demethyldiltiazem in $100 \mathrm{ml}$ methanol. The standard curve was prepared by spiking drug free plasma with a known weight of diltiazem and $\mathrm{N}$-demethyldiltiazem at concentration levels of 5, 10, 25, 50, 100 and 200 $\mathrm{ng} / \mathrm{ml}$. The standard plasma samples were stored at $-20^{\circ}$ in glass bottles.

\section{Extraction Procedure}

A $1.0 \mathrm{ml}$ aliquot of the plasma was accurately measured into a glass tube with a Teflon lined screw cap, followed by addition of $50 \mu \mathrm{l}$ of internal standard (4 $\mu \mathrm{g} / \mathrm{ml}$ of verapamil $\mathrm{HCl}$ in aqueous solution and 4.0-ml mixture of diethyl ether and n-hexane (1:1). The mixture was vortexed for $1 \mathrm{~min}$ using a vortex mixer and then centrifuged at 3500 rpm for 10 minutes. The upper organic layer was transferred into a reactivial (Pierce Reacti-vial, USA) and then evaporated to dryness at $40^{\circ}$ under a gentle stream of nitrogen gas.
The residue was reconstituted with 75 $\mu l$ of mobile phase and $50 \mu \mathrm{l}$ injected into the column.

\section{Assay Validation}

Samples were quantified using height ratio of diltiazem or $\mathrm{N}$ demethyldiltiazem over the internal standard. Extraction recovery, withinday and between-day precision and accuracy studies $(n=6)$ of the method were carried out using plasma standard samples. The recovery of the extraction procedure for diltiazem, $\mathrm{N}$ demethyldiltiazem and internal standard were calculated by comparing the peak height obtained after extraction with that of aqueous solutions of corresponding concentrations without extraction. The accuracy was expressed as percentage error, obtained by calculating the percentage of difference between the measured and the spiked concentration over that of the spiked value, whereas the precision was denoted using the coefficient of variation.

\section{RESULTS AND DISCUSSION}

Diltiazem and its metabolites are weakly basic amines and the amino groups are known to interact strongly with the stationary support, causing peak tailing and broadening. ${ }^{12 \& 13}$ Some short-chain tertiary amine modifiers such as triethylamine (TEA) are very effective in improving peak symmetry and reducing retention of solutes with amino functional groups. ${ }^{14}$ Therefore, TEA 
in $0.08 \%$ was incorporated into the mobile phase to improve the resolution as well as peak symmetry of diltiazem and its metabolite.

Chromatograms obtained with blank plasma and plasma sample of a healthy volunteer after $4 \mathrm{hr}$ dosing with $90 \mathrm{mg}$ diltiazem are shown in Figs. 2A and 2B. The retention times of N-demethyldiltiazem, diltiazem $\mathrm{HCl}$ and internal standard (verapamil $\mathrm{HCl}$ ) were $4.98, \quad 5.78$ and 9.88 minutes respectively. The blank sample was clean and no interfering peak was observed at the retention times of the above mentioned components.

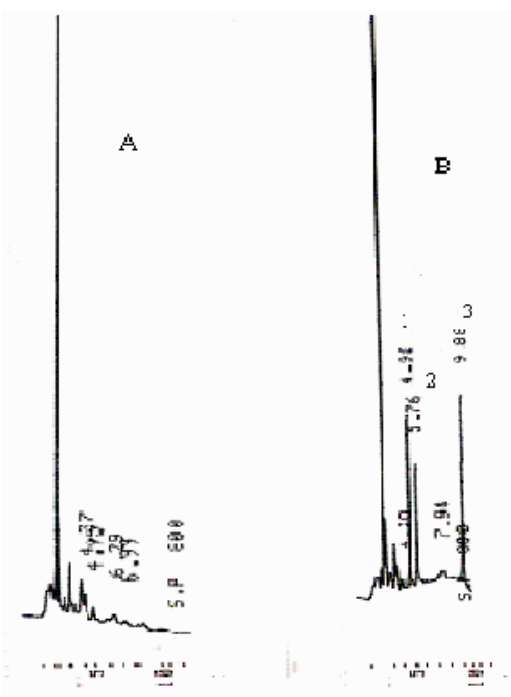

Fig. 2: Chromatograms of (A) blank plasma and (B) plasma sample obtained from a volunteer at 4 hours after oral administration of $90 \mathrm{mg}$ controlled release diltiazem showing peaks for N-demethyldiltiazem (1), diltiazem (2) and the internal standard, verapamil (3).
The extraction recovery of diltiazem was determined by comparing the peak height obtained by direct injection of standard aqueous solutions to those obtained after the plasma extraction procedure. A mixture of diethyl ether and nhexane $(1: 1 \quad \mathrm{v} / \mathrm{v})$ gave better recoveries for $\mathrm{N}$-demethyldiltiazem, diltiazem and internal standard as well as provided cleaner chromatograms compared to using $\mathrm{n}$ hexane, diethyl ether, methyl tertiary butyl ether, ethyl acetate or chloroform alone as the extracting solvent. The recovery of all the components was more than $90 \%$.

The mean standard curve of diltiazem only $(n=6)$ is shown in Fig. 3. A linear correlation was found between the peak height ratio of diltiazem and the internal standard versus diltiazem concentration in the plasma in the range of $5-200 \mathrm{ng} / \mathrm{ml}$ with a determination coefficient of 0.9995. Similarly, linear correlation was observed between the peak height ratio of $\mathrm{N}$-demethyldiltiazem and verapamil versus plasma $\mathrm{N}$ demethyldiltiazem concentration in the range of 5-200 ng/ml.

Within day and between day precision and accuracy of the method assessed by analysis of plasma samples at various concentration levels of diltiazem and Ndemethyldiltiazem are separately given in Tables 1 and 2 respectively. The values for the coefficient of variation of both within day and between day were all less than $10 \%$ at the concentration range determined 


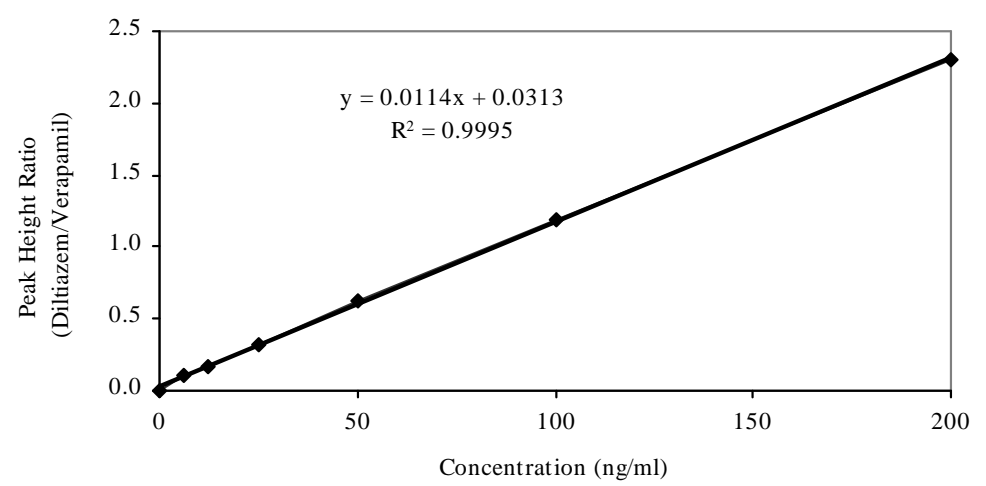

Fig. 3: Mean standard curve of Diltiazem $\mathrm{HCl}$

Table 1: Within day and between day precision and accuracy of Diltiazem $\mathrm{HCl}$ $(n=6)$.

\begin{tabular}{||c|c|c|c|c|c|c||}
\hline \hline Concentration & \multicolumn{2}{|c|}{ Recovery } & \multicolumn{2}{c|}{ Within day } & \multicolumn{2}{c||}{ Between day } \\
\hline$(\mathrm{ng} / \mathrm{ml})$ & \multicolumn{2}{|c|}{$(\mathrm{ng} / \mathrm{ml})$} & $\begin{array}{l}\text { Precision } \\
(\mathrm{CV} \%)\end{array}$ & $\begin{array}{l}\text { Accuracy } \\
(\% \text { error })\end{array}$ & $\begin{array}{l}\text { Precision } \\
(\mathrm{CV} \%)\end{array}$ & $\begin{array}{l}\text { Accuracy } \\
(\% \text { error })\end{array}$ \\
\hline 5 & 5.10 & \pm 0.26 & 5.10 & +2.0 & 6.48 & +8.0 \\
\hline 10 & 10.30 & \pm 0.40 & 3.88 & +3.0 & 8.25 & -3.0 \\
\hline 25 & 25.07 & \pm 1.39 & 5.54 & +0.3 & 6.06 & -0.4 \\
\hline 50 & 50.67 & \pm 1.07 & 2.11 & +1.3 & 3.27 & +2.2 \\
\hline 100 & 101.50 & \pm 1.87 & 1.84 & +1.5 & 1.65 & +3.2 \\
\hline 200 & 203.77 & \pm 1.26 & 0.62 & +1.9 & 0.72 & -2.6 \\
\hline
\end{tabular}

$\mathrm{CV}=$ Coefficient of Variation \pm Standard deviation.

Table 2: Within day and between day precision and accuracy of $\mathrm{N}$ Demethyldiltiazem $(n=6)$.

\begin{tabular}{|c|c|c|c|c|c|c|}
\hline \multirow{2}{*}{$\begin{array}{l}\text { Concentration } \\
(\mathrm{ng} / \mathrm{ml})\end{array}$} & \multirow{2}{*}{\multicolumn{2}{|c|}{$\begin{array}{c}\text { Recovery } \\
(\mathrm{ng} / \mathrm{ml})\end{array}$}} & \multicolumn{2}{|c|}{ Within day } & \multicolumn{2}{|c|}{ Between day } \\
\hline & & & $\begin{array}{c}\text { Precision } \\
\text { (CV \%) }\end{array}$ & $\begin{array}{l}\text { Accuracy } \\
\text { (\% error) }\end{array}$ & $\begin{array}{c}\text { Precision } \\
(\mathrm{CV} \%)\end{array}$ & $\begin{array}{l}\text { Accuracy } \\
\text { (\% error) }\end{array}$ \\
\hline 5 & 4.97 & \pm 0.38 & 7.64 & -6.0 & 8.18 & +10.0 \\
\hline 10 & 9.67 & \pm 0.40 & 4.14 & -3.3 & 8.54 & +3.0 \\
\hline 25 & 25.17 & \pm 0.71 & 2.82 & +0.7 & 3.79 & -2.8 \\
\hline 50 & 50.10 & \pm 1.04 & 2.08 & +0.2 & 2.37 & -2.2 \\
\hline 100 & 101.97 & \pm 3.12 & 3.06 & +2.0 & 2.58 & +2.9 \\
\hline 200 & 202.93 & \pm 3.35 & 1.65 & +1.5 & 1.56 & -2.8 \\
\hline
\end{tabular}

$\mathrm{CV}=$ Coefficient of Variation \pm Standard deviation 
for both diltiazem and Ndemethyldiltiazem, whereas the percentage error were within $10 \%$. It is interesting to note that within day and between day $\mathrm{CV}$ values for both diltiazem and $\mathrm{N}$-demethyldiltiazem appeared to be similar suggesting that the precision as well as accuracy was not compromised during between-day analysis. The limit of detection was approximately $2.5 \mathrm{ng} / \mathrm{ml}$ at a signalto-noise ratio of $3: 1$. However, the limit of quantification was set at 5 $\mathrm{ng} / \mathrm{ml}$ being the lowest concentration used in the construction of the standards curve. But in the reported study, quantification of diltiazem and other metabolites was found to be set at $10 \mathrm{ng} / \mathrm{ml}^{15}$ In most of the reported methods, diltiazem alone or along with deacetyldiltiazem have been quantified using HPLC $^{16}$ or gas chromatography. ${ }^{17}$ Whilst a fewer methods have been used to quantify diltiazem, N-demethyldiltiazem and other less common metabolites simultaneously in the spiked plasma or animal plasma ${ }^{18}$ and patient plasma for monitoring steady state concentrations. $^{11 \& 16}$ Moreover, these methods have been utilized to lesser extent for quantification of diltiazem and $\mathrm{N}$-demethyldiltiazem in healthy human plasma after single oral administration of controlled release tablets or pellets. Therefore, the method developed in this study was applied in a pharmacokinetic study of six normal healthy volunteers on single dosage of sustained release pellets containing diltiazem $\mathrm{HCl} 90$ mg. Fig. 4 shows the mean plasma profiles of both diltiazem and $\mathrm{N}$ demethyldiltiazem following a morning oral dose of diltiazem $\mathrm{HCl}$. It is evident that the plasma concentration of diltiazem was less than $\mathrm{N}$-demethyldiltiazem due to its first pass effect and both could still be detected after $24 \mathrm{hr}$ dosing.

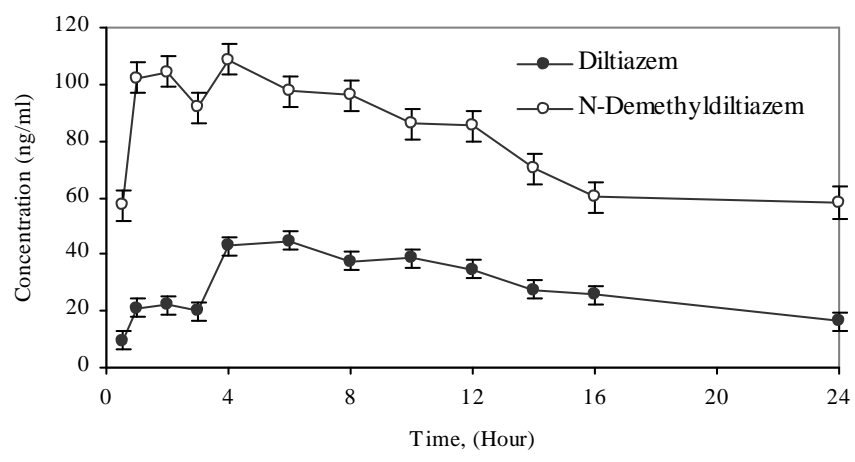

Fig. 4: Mean plasma diltiazem and N-demethyldiltiazem concentrations following administration of sustained release diltiazem pellets. Mean \pm SEM (vertical bar), $n=6$. 


\section{Conclusion}

The present HPLC method is sensitive, simple, specific and appropriate to be used for determination of plasma diltiazem and its major metabolite, $\mathrm{N}$-demethyldiltiazem in pharmacokinetic/ bioavailability studies. The $\mathrm{pH}$ of the mobile phase used was well within the practical working $\mathrm{pH}$ range of common reversed phase $\mathrm{C} 18$ columns, which is typically between $\mathrm{pH} 3-7$, thus avoiding the use of more expensive $\mathrm{pH}$ sensitive columns. Moreover, the total analysis time for assay method was less as compared to the reported methods.

\section{REFERENCES}

1- M. Chaffman and R. N. Brogden, Drugs 29, 387 (1985).

2- P. Hoglund and L.G. Nilsson, Ther. Drug Monit., 11, 558 (1989).

3- H. Christensen, E. Carlson, A. Asberg, L. Schram and KJ. Berg, J. Chromatogr. B Biomed. Appl., 657, 133 (1994).

4- V. Ascalone, M. Locatelli and B. Malavasi, ibid., 17, 522 (2003).

5- P. K. Yeung, T. J. Montague, B. Tsui and C. McGregor, Anal. Bioanal. Chem., 376, 848 (2003).
6- R. S. Chaudhary, S. S. Gangwal, M. K. Avachat, Y. N. Shah and K. C. Jindal, J. Chromatogr., 614, 261 (1993).

7- D. R. Abernethy, J. B. Schwartz and E. L. Todd, ibid., 342, 216 (1985).

8- R. Boulieu, J. L. Bonnefous and S. Ferry, ibid., 528, 542 (1990).

9- C. D. Kinney and J. G. Kelly, ibid., 382, 377 (1986).

10- Ascalone and L. Dalbo, ibid., 423, 239 (1987).

11- S. C. Montamat, D.R. Abernethy and J. R. Mitchell, ibid., 415, 203 (1987)

12- K. E. Biji, Cs. Horvath, W.R. Melander and A. Nahum, ibid., 203, 65 (1981).

13- A. Nahum and Cs. Horvath, ibid., 203, 53 (1981).

14- J. S. Keil, S. L. Morgan and R.K. Abramson, ibid. 320, 313 (1985).

15- A. Zarghi, S. Dadashzadeh and A. J. Ebrahimian, Pharm. Pharmacol. Commun., 6, 341 (2000).

16- L. M. Dube. N. Mousseau and I. J. Mcgilvery, J. Chromatogr., 430, 103 (1988).

17- O. Grech-Belanger, E. Leboeuf and S. Langlois, ibid., 417, 89 (1987).

18- K. J. Goebel and E. U. Kolle, ibid., 345, 355 (1985). 